\title{
Lamotrigine Induced Severe Cutaneous Reaction
}

\author{
Roka YB ${ }^{1}$, Roka N², Adhikari HB ${ }^{3}$ \\ ${ }^{1}$ Dr. Yam B. Roka, MBBS, MS, MCh. Chief of Neurosurgery, Neuro Hospital, Biratnager, ${ }^{2}$ Dr.Narayani Roka, MBBS, MS, Chief \\ Ophthalmologist, Golcha Eye Hospital, Biratnagar, ${ }^{3}$ Dr. Hari Bikram Adhikari, MBBS, Medical Officer Neuro Hospital, \\ Biratnagar, Nepal.
}

Address for correspondence: Dr. Yam B Roka, E-mail: dryamroka@yahoo.com

\begin{abstract}
Antiepileptic drugs are used commonly by physicians, neurologist and neurosurgeons in Nepal. Lamotrigine is an effective antiepileptic drug used as an add-on and monotherapy for a variety of seizure types in both adults and children. Rashes due to AED including SJS and hypersensitivity syndrome leading to hospitalization occur in approximately $0.33 \%$ of adults and $0.8 \%$ in children. Reactions due to LTG alone occur in around $5.7 \%$ of cases. We report probably the first case of this reaction in a 12-year old girl with a brief discussion on its etiology and management.
\end{abstract}

Key words: AED, Epilepsy, Lamotrigine, Skin rash, Stevens-Johnson syndrome.

\section{Introduction}

A ntiepileptic drugs (AED) are used commonly by physicians, neurologist and neurosurgeons in Nepal. Although rare, cutaneous reactions like Stevens-Johnson syndrome (SJS), toxic epidermal necrosis (TEN), and drug rash with eosinophilia and systemic symptoms (DRESS) have been associated with this drug ${ }^{1,2,3}$. Lamotrigine (LTG) is an effective antiepileptic drug used as an add-on and monotherapy for a variety of seizure types in both adults and children. Although this drug was available for a long time in other countries it has been recently introduced in Nepal. Allergic skin reaction due to AED occurs usually in the first 8 weeks in approximately $10 \%$ of patients ${ }^{4}$. Rashes due to AED including SJS and hypersensitivity syndrome leading to hospitalization, occur in approximately $0.33 \%$ of adults and $0.8 \%$ in children ${ }^{4,5}$. Reactions due to LTG alone occur in around $5.7 \%$ of cases $^{6}$. We report probably the first case with a brief discussion on its etiology and management.

\section{The Case}

A 12-year old girl presented with history of recurrent attacks of loss of consciousness associated with tongue biting, frothing and jerky hand movements for the past 4 months. She had ten of such episodes that lasted from 1-5 mins and postictal drowsiness for 3-4 hours. Although she had been started on phenytoin there was no control of the seizures. There was no family history of seizures. No other history being significant she was advised for an electroencephalogram (EEG) and computed tomogram (CT) of the head. The EEG showed features of generalized seizures while the CT was normal, suggesting Idiopathic generalized epilepsy. Rest of the hematology reports was normal. She was diagnosed as refractory epilepsy and hence started on LTG with a starting dose of $50 \mathrm{mg}$ once daily for 5 days and then continued on 100mg daily. On her first followup at 31 days she had no attacks, was doing fine and hence asked for 5-monthly follow up.

She then presented on the $42^{\text {nd }}$ day of therapy with history of rashes all over the body, itching, swelling of the limbs and itching of eyes. She also had rashes on her lips, arms and acral areas (Figure 1).

Dermatology and eye consultation confirmed the diagnosis as SJS. She was started on oral prednisolone in tapering dose, mouth wash, antipruritics and eye drops. Her itching improved on the $2^{\text {nd }}$ day and the rashes disappeared over 5 days. LTG was discontinued and she was started on low escalating dose of sodium valproate. She was discharged on the $6^{\text {th }}$ day and on her follow up after 2 months she is asymptomatic with no plans for a rechallenge. 

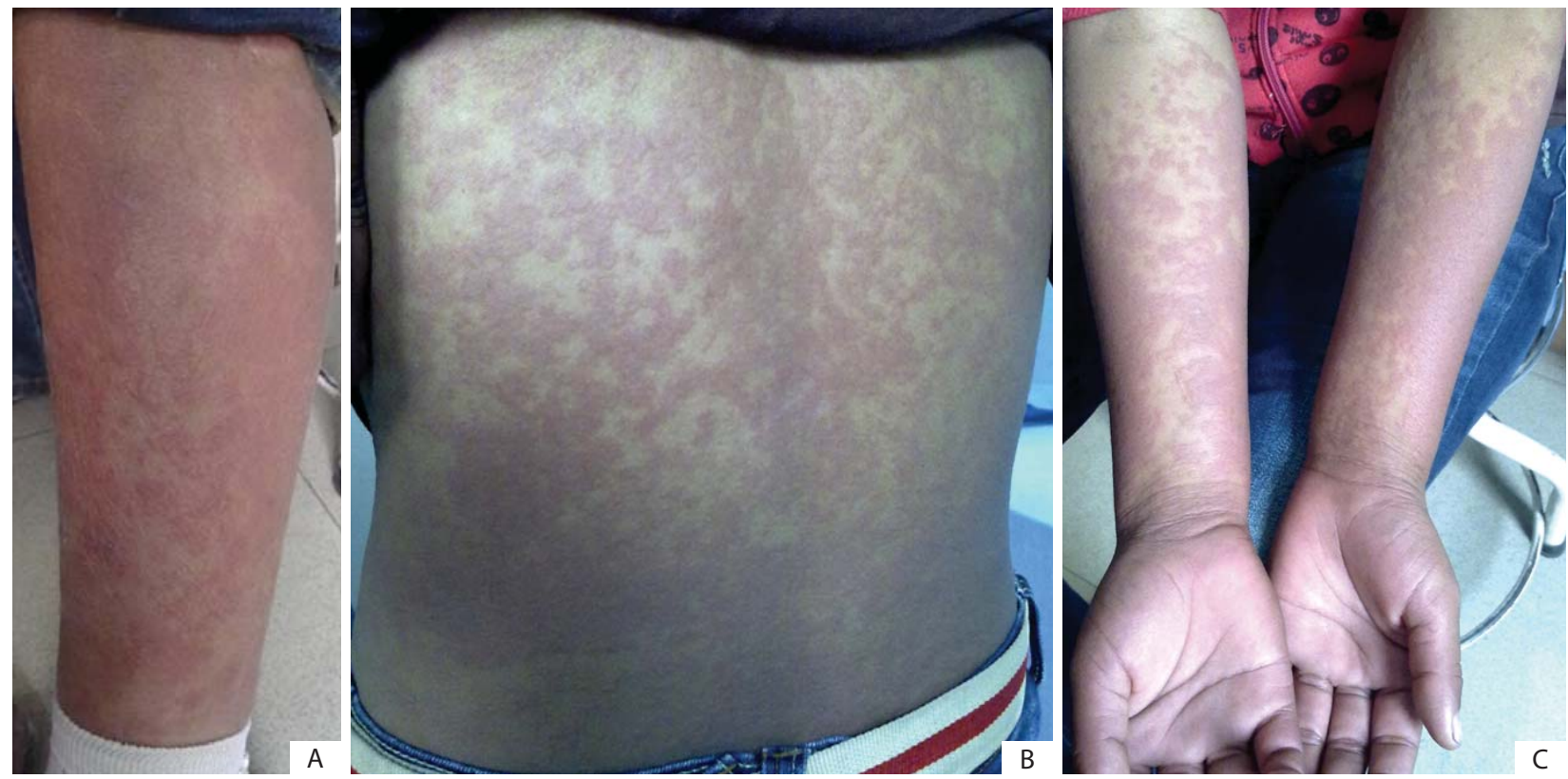

Fig 1: Clinical photograph showing rashes over the leg (A), trunk (B) and hands (C).

\section{Discussion}

Epilepsy in Nepal is treated by physicians, neurologist, neurosurgeons, pediatricians and also as over the counter drug by other health related professionals. LTG has recently been launched by several companies and the need to understand its adverse effects are must to prevent complications to the patient. LTG is presently used for treatment of pediatric patients with both partial onset and primary generalized seizures refractory to the major older antiepileptic drugs. SJS and other cutaneous reactions are associated with several AEDs including LTG, carbamazepine, phenytoin and valproate. This group specific cross-sensitivity among AED may be explained by the "hapten hypothesis," which states that common metabolic and immunologic pathways are responsible for the rashes caused by these drugs. This reaction although rare can be limited to the skin or involve other visceral organs like the liver, pancreas, in which case it can be life threatening ${ }^{1-6}$. AED can also be associated with a triad of fever, skin reactions and internal organ involvement which is called as antiepileptic hypersensitivity syndrome ${ }^{7,8}$. LTG is chemically unrelated to other AED but is as effective in epilepsy when used as monotherapy or in combination. Skin reactions due to LTG occur in less than $1 \%$ and may depend on the rate of escalation, hypersensitivity reaction, female gender, ethnicity, age less than 13 years, initial strength of dose and recently associated HLA$B^{*} 1502^{7,8}$.

In a study of AED toxicity in Asians from Taiwan it was found that carbamazepine, phenytoin and LTG were the major causes of skin reaction with mortality of $6.1 \%^{1}$
In a study in Chinese adults with epilepsy it was found that the drugs were tolerated well ${ }^{9}$. Although these studies show that Asians do tolerate AED well there has yet to be a study conducted in Nepal in this aspect. Occurrence of AED adverse reaction needs immediate drug discontinuation, steroids and investigations to rule out visceral organ involvement. Eye and mucous care may need special attention. Usually the trend is to change over to other AED excluding carbamazepine and phenytoin. Recently many papers have confirmed the safety of rechallenge with LTG after the initial symptoms have subsided. In a prospective series of such cases a classification system was used to rate the severity of LTG rashes and a pooled meta-analysis done to assess the severity on rechallenge (Table-1). For groups with score of 0 , drug reduction was advised, for moderate rashes (rated 1-2) rechallenge was possible with careful observation ${ }^{10}$. The rechallenge was safer if it was done after 4 weeks of resolution of the rash and in slowly escalating drug dosages.

Table 1: Rating scale for dermatological drug eruptions.

\begin{tabular}{|l|c|c|}
\hline \multicolumn{1}{|c|}{ Clinical Feature } & Present & Absent \\
\hline Purpura, tenderness or blistering & 3 & 0 \\
\hline Exfoliation or erythroderma & 1 & 0 \\
\hline $\begin{array}{l}\text { Facial or mucous membrane } \\
\text { involvement }\end{array}$ & 1 & 0 \\
\hline Lymphadenopathy & 1 & 0 \\
\hline $\begin{array}{l}\text { Hematological abnormalities or } \\
\text { elevated transaminases }\end{array}$ & 1 & 0 \\
\hline $\begin{array}{l}\text { Constitutional symptoms (fever, } \\
\text { malaise etc) }\end{array}$ & 1 & 0 \\
\hline
\end{tabular}




\section{Conclusion}

LTG was recently launched in Nepal and is effective in partial onset and primary generalized seizures refractory to other medications in children. Skin rash is a potential complication that needs to be known by all the attending doctors to prevent further morbidity and even mortality.

\section{References}

1. Yang $\mathrm{CY}$, Dao RL, Lee TJ, Lu CW, Yang CH, Hung Sl, Chung WH. Severe cutaneous adverse reactions to antiepileptic drugs in Asians. Neurology 2011 6:2025-33.

2. An DM, Wu XT, Hu FY, Yan B, Stefan H, Zhou D. Association study of lamotrigine-induced cutaneous adverse reactions and HLA-B*1502 in a Han Chinese population. Epilepsy Res 2010 92:226-30.

3. Roquin G, Peres M, Lerolle N, Dib N, Mercat A, Croue A, Augusto JF. First report of lamotrigine-induced drug rash with eosinophilia and systemic symptoms syndrome with pancreatitis. Ann Pharmacother 2010 44:1998-2000.

4. Guberman AH, Besag FM, Brodie MJ, Dooley JM, Duchowny MS, Pellock JM, Richens A, Stern RS, Trevathan E. Lamotrigine-associated rash: risk/ benefit considerations in adults and children. Epilepsia 1999 40:985-91.

5. Seo HJ, Chiesa A, Lee SJ, Patkar AA, Han C, Masand PS, Serretti A, Pae CU. Safety and tolerability of lamotrigine: results from 12 placebo-controlled clinical trials and clinical implications. Clin Neuropharmacol 2011 34:39-47.

6. Hirsch LJ, Weintraub DB, Buchsbaum R, Spencer HT, Straka T, Hager M, Resor SR Jr. Predictors of Lamotrigine-associated rash. Epilepsia 2006 47:31822.

7. Schlienger RG, Shapiro LE, Shear NH. Lamotrigineinduced severe cutaneous adverse reactions. Epilepsia 199839 7:S22-6.

8. An DM, Wu XT, Hu FY, Yan B, Stefan H, Zhou D. Association study of lamotrigine-induced cutaneous adverse reactions and HLA-B*1502 in a Han Chinese population. Epilepsy Res 2010 92:226-30.

9. Zeng K, Wang X, Xi Z, Yan Y. Adverse effects of carbamazepine, phenytoin, valproate and lamotrigine monotherapy in epileptic adult Chinese patients. Clin Neurol Neurosurg 2010 112:291-5.

10. Aiken $C B$, Orr $C$. Rechallenge with lamotrigine after a rash: a prospective case series and review of the literature. Psychiatry 2010:27-32.

\section{How to cite this article?}

Roka YB, Roka N, Adhikari HB. Lamotrigine Induced Severe Cutaneous Reaction. J Nepal Paediatr Soc 2012;32(2):172-174. 